\title{
Actin, microtubule, septin and ESCRT filament remodeling during late steps of cytokinesis
}

Cyril Addi ${ }^{1,2,3, \#, ~ J i a n ~ B a i}{ }^{1,2,3, \#}$ and Arnaud Echard ${ }^{1,2}$

1 Membrane Traffic and Cell Division Lab, Cell Biology and Infection department Institut Pasteur, 25-28 rue du Dr Roux, 75724 Paris cedex 15, France

2 Centre National de la Recherche Scientifique CNRS UMR3691, 75015 Paris, France 3 Sorbonne Universités, Université Pierre et Marie Curie, Université Paris 06, Institut de formation doctorale, 75252 Paris, France

\# equal contribution, alphabetical order

correspondence: arnaud.echard@pasteur.fr 


\section{ABSTRACT}

Cytokinesis is the process by which a mother cell is physically cleaved into two daughter cells. In animal cells, cytokinesis begins with the contraction of a plasma membraneassociated actomyosin ring that is responsible for the ingression of a cleavage furrow. However, the post-furrowing steps of cytokinesis are less understood. Here, we highlight key recent findings that reveal a profound remodeling of several classes of cytoskeletal elements and cytoplasmic filaments (septins, microtubules, actin and ESCRT) in the late steps of cytokinesis. We review how this remodeling is required first for the stabilization of the intercellular bridge connecting the daughter cells and then for the steps leading up to abscission. New players regulating the abscission (NoCut) checkpoint, which delays abscission via cytoskeleton and ESCRT remodeling in response to various cytokinetic stresses, will also be emphasized. Altogether, the latest discoveries reveal a crucial role for posttranslational modifications of the cytoskeleton (actin oxidation, septin SUMOylation) and an unexpected requirement of ESCRT-III polymer dynamics for successful abscission. 
Actin polymerization and turnover are essential for furrow ingression in animal cells $[1,2]$. After furrow ingression, a microtubule (MT)-filled intercellular bridge connects the daughter cells for several hours before the final cut [3]. Ten years ago, a breakthrough in the field was the finding of a role for the Endosomal Sorting Complex Required for Transport (ESCRT) in cytokinetic abscission, which at that time was known for intraluminal vesicle scission in late endosomes/multivesicular bodies (MVBs) and for retroviral budding [4-6]. Although the detailed mechanism is under debate [7], the current literature indicates that the ESCRT machinery is sufficient for outward membrane budding and fission [8], an event that is topologically equivalent to cytokinesis. In this review, we will focus on 1) how the septin cytoskeleton is regulated to maintain bridge stability, 2) how ESCRT polymers are turnedover to promote abscission and 3 ) the recent findings regarding MT and F-actin clearance within the bridge prior to abscission to allow for correct ESCRT filament assembly.

\section{Septin filament dynamics and intercellular bridge stability}

The stability of the cytokinetic bridge becomes independent of actin filaments soon after furrow ingression but relies on septins [9-11]. Septins are GTP-binding proteins that assemble into non-polar, rod-shaped oligomeric complexes and higher-order structures such as filaments and bundles [12]. Septins localize to the intercellular bridge and their inactivation usually leads to binucleated cells due to either an unstable, and sometimes displaced, cleavage furrow or intercellular bridge relaxation in several animal models [1316], but not in C. elegans [17]. In Drosophila S2 cells, the septin Peanut (Septin 7) acts in complex with anillin to maintain connections between the midbody and the plasma membrane, thus maintaining furrow/bridge stability. In addition, septins are required for trimming away excess anillin-positive membranes from the early bridge, which promotes its maturation, both in Drosophila [11] and human [16] cells. Among human septins, SEPT9 plays a distinct and later role in cytokinesis, since its inactivation essentially delays abscission but does not generate binucleated cells $[14,16,18]$. Altogether, recent evidence indicates a role for septins in bridge maturation, a step preceding and required for ESCRT-III recruitment to the abscission site $[16,17]$, but the exact mechanistic roles of septins in this process are not yet fully understood. 
In yeast, septins were the first substrates reported to be post-translationally modified by SUMOylation (a moiety resembling Ubiquitin), but this modification is not critical for cytokinesis $[19,20]$. In contrast, findings in human cells revealed this year that septins are SUMOylated and that this modification is important for cytokinesis [21]. Indeed, nonSUMOylable mutant septins assemble in abnormally long and thick bundles that do not turnover. These bundles likely constitute a physical block within the intercellular bridge, which explains bridge relaxation and observed binucleated cells [21]. Whether SUMO directly controls filament assembly into higher-order structures or whether SUMOylation of septins regulates the interaction of proteins that inhibit the formation of large bundles remains to be investigated. In any case, this highlights a new role for SUMOylation both in cytoskeletal dynamics and cytokinesis (Figure 1A).

\section{ESCRT-III filaments and cytokinetic abscission}

As mentioned in the introduction, current models indicate that the ESCRT machinery is responsible for the final abscission, which takes place on the midbody side (also called the "secondary ingression site") [10,16,22-24]. Consistently, depletion of several key ESCRT components or associated proteins such as ALIX, TSG101 (ESCRT-I) and several CHMPS (ESCRT-III) leads to cytokinetic delay, abscission failure and binucleated cells in human cells $[4,5,10]$. Mechanistically, ESCRT-III can polymerize into filaments in vitro [7] and the presence of $17 \mathrm{~nm}$ cortical intertwined filamentous helices have been observed at the abscission site in human cells by electron and X-ray microscopy $[10,25,26]$. In cells depleted for the ESCRT-III component CHMP2A, these helices are no longer present, and concomitantly no cortical constriction is observed [10]. This indicates that either these helices are made of ESCRT, or the ESCRT machinery is required for these filaments to polymerize and constrict the intercellular bridge. This is a crucial point that has to be resolved in our standard model of abscission, perhaps by combining super-resolution and EM tomography. Interestingly, other filamentous structures made of anillin and septins are also observed at the secondary ingression site before CHMP4B recruitment [16]. Thus, the relationship between these different filamentous polymers and the helices observed by EM has to be clarified in future studies. Furthermore, published micrographs show ESCRT-III- 
dependent helices in bridges constricted to no less than 150-200 nm [10,26]. Thus, more work is needed to observe helices in further constricted states in order to definitively prove that ESCRT-III-dependent helices drive the final pinch. This might prove tedious if the final constriction step is fast and thus difficult to catch. A provocative, alternative possibility would be that after ESCRT-III constriction to $150-200 \mathrm{~nm}$, there is an additional ESCRTindependent step leading to the final scission of the intercellular bridge at 3-10 nm, perhaps involving lipid modifications $[27,28]$.

Recent findings clarified the mechanisms by which the ESCRT-III machinery is recruited to the intercellular bridge. It was shown that Cep55 plays a pivotal role in directly recruiting TSG101 and ALIX to the midbody in mammalian cells $[5,29]$. It is now shown that ALIX and TSG101 act in parallel to fully recruit ESCRT-III components [30]: ALIX is activated by phosphorylation [31] and directly interacts with CHMP4B, whereas the ESCRT-I components TSG101 and VPS28 recruit ESCRT-II proteins which in turn recruit the ESCRT-III CHMP4B. Yet, these two parallel pathways are not equivalent, as only ALIX appears to have a specific role in the prevention of binucleation [5,30]. Similarly, CHMP4C, another ESCRT protein involved in the abscission checkpoint (see below), is recruited via ALIX but not TSG101 [30]. A question that remains poorly understood is how ESCRT-III components, initially found at the midbody are later found at the abscission site. Does this involve a separate recruitment from a cytosolic pool? Does ESCRT-III physically translocate from the midbody to the abscission site? Is this related to relaxation of tension within the intercellular bridge [32,33]? Despite these interrogations, it is known that the relocalization of CHMP4B from the midbody to the abscission site depends on anillin/septins [16], as well as F-actin clearance (see below).

Regarding the issue of the spatial and temporal recruitment of the ESCRT machinery at the midbody and the abscission site, important progress has been made this year. Nearendogenous expression levels of tagged proteins combined with fluorescent microscopy revealed that the AAA-ATPase VPS4, which is known to disassemble ESCRT filaments [7], does not arrive to the midbody just before abscission as initially thought [22], but instead is continuously present throughout ESCRT-III subunit recruitment [26]. The presence of VPS4 at the midbody explains why ESCRT-III filaments are highly dynamic (their different subunits display a high turnover, with residence time of $20 \mathrm{sec}$ ) [26]. At first counter intuitively, this 
process leads to a net increase in the amount of CHMP4B during progress toward abscission. Indeed, depletion of the AAA-ATPase VPS4 abolishes CHMP4B turnover at the midbody, diminishes the recruitment of ESCRT-III, prevents the constriction of the intercellular bridge, and results in a strong delay in abscission [26]. Remarkably, in vitro reconstitution assays coupled with high-speed atomic force microscopy (AFM) revealed that ESCRT-III filaments form spirals that undergo both rapid growth and shrinkage in the presence of VPS4 and ATP [26]. Altogether, this suggests that continuous VPS4-dependent turnover of ESCRT-filaments is crucial for filament growth and abscission in vivo, revisiting the mechanistic role of VPS4 and ATP hydrolysis in polymer constriction. Interestingly, the spiral helices could not fully contract in vitro [26]. Thus, either an additional component is missing for full constriction, or, as mentioned above, the ESCRT machinery is required for successful abscission but does not drive it to completion. Importantly, turnover of ESCRT-III by Vps4 could be a general principle in ESCRT-dependent mechanisms since a continuous and stochastic recruitment of Vps4 throughout the process of intraluminal vesicle budding in yeast MVBs has now been observed [34]. Understanding how polymer remodeling translates into fission is thus a major challenge for the future.

Microtubule and F-actin clearance at the abscission site

Microtubule bundles are locally severed at the secondary ingression site $[10,16,22,23]$ and this is presumably an important step for ESCRT filaments to be able to constrict to completion. Remarkably, the MT-severing AAA-ATPase spastin directly interacts with the ESCRT-III component CHMP1B, and is required for normal abscission [10, 33, 35,36]. This suggests a molecular mechanism coordinating ESCRT recruitment and MT clearance at the abscission site. The same coordination between ESCRT-III and spastin has also been recently observed during nuclear envelope reformation at mitotic exit [37]. However, the importance of spastin for severing MTs at the abscission site is discussed, since spastin-independent, buckling-induced MT severing has also been proposed [38].

In addition to MTs, F-actin must be cleared from the intercellular bridge for successful abscission $[23,39,40]$. However, whether this has to occur specifically at the secondary site 
or all along the bridge has not yet been resolved. Two mechanisms for F-actin clearance have been elucidated. The first mechanism relies on the Rab35 GTPase which recruits an effector, the PtdIns(4,5) $P_{2}$ lipid phosphatase OCRL [41], to the intercellular bridge [39]. Ptdlns $(4,5) P_{2}$ hydrolysis by OCRL limits F-actin oligomerization and is required for normal abscission [28,39]. In addition, Rab11-FIP3-positive endosomes deliver the p50RhoGAP cargo to the bridge to further limit Rho GTPase activation and thus F-actin polymerization in bridges [23]. The second mechanism, also depending on Rab35, was revealed this year and depends on another effector of this GTPase: the oxidoreductase MICAL1 [40] (Figure 1B). MICAL1 is an enzyme that oxidizes methionine residues on F-actin and induces filament depolymerization in vitro [40,42] (for a recent review regarding MICAL enzymes see [43]). Rab35 binding to MICAL1 is sufficient to activate the enzymatic activity of MICAL1, which is tightly regulated [40]. In addition, Rab35 recruits a pool of MICAL1 at or close to the abscission site a few minutes before abscission [40]. Altogether, Rab35/MICAL1 actively clear F-actin from bridges, which is a step required for normal abscission and recruitment of ESCRT-III to the abscission site [40]. Interestingly, other proteins that need to be discovered also contribute to MICAL1 recruitment during cytokinesis, and may include other Rab GTPases known to interact with MICAL1 [43]. In summary, two GTPases control parallel pathways that cooperate in F-actin clearance and correct ESCRT-III recruitment at the abscission site. On the one hand, these pathways limit the amount of F-actin polymerization via Rab35/OCRL1 and Rab11/MICAL1 and, on the other hand, actively depolymerize F-actin at the bridge via Rab35/MICAL1. It remains to be established how Rab35 is activated at the midbody and the abscission site, and whether vesicular delivery is involved in enriching this GTPase at specific locations within the intracellular bridge (for a recent review on membrane traffic and cytokinesis, see [44]). Another interesting issue is to determine whether Rab11 and Rab35 act sequentially or in parallel during the process of F-actin clearance.

\section{Conclusion and open questions}

Several recent studies have helped to understand the multiple events of cytoskeletal remodeling involved in the late steps of cytokinesis (Figure 2). A number of remaining questions regarding the remodeling of septins, MTs, actin and ESCRT filaments have been 
detailed above and the main ones are summarized in the BOX. Besides answering these questions, several areas are expected to yield exciting findings in future studies.

First, the study of cytokinesis in abnormal or pathological situations, such as during aging or when a stress is present. An abscission checkpoint, also called the "abscission checkpoint", was discovered several years ago in seminal works in yeast (termed the "NoCut checkpoint" in this organism) [45] and in mammalian cells [46]. This evolutionarily-conserved checkpoint delays abscission when lagging chromatin abnormally stays in the intercellular bridge, but also after a variety of other cytokinetic stresses such as defects in nuclear pore reformation or high levels of intercellular tension [33,47-50]. Mechanistically, activated Aurora B mediates the checkpoint by phosphorylating the ESCRT-III subunit CHMP4C, which acts as a negative regulator of ESCRT-III filament assembly [51-53]. In addition, the ATPase VPS4 interacts with the checkpoint regulator ANCHR, which together with CHMP4C retains VPS4 at the midbody and thus delays abscission [54]. The kinase ULK3 also phosphorylates and inhibits IST1, which is an ESCRT-III component required for abscission and a key regulator of Vps4 [55]. Furthermore, recent work identified the CDK-like kinases Clk1, Clk2 and Clk4 as additional activators of Aurora B [56]. Altogether, there is clear evidence that the Aurora Bdependent abscission checkpoint regulates the assembly and constriction of ESCRT-III filaments in response to cytokinetic stresses. Interestingly, activation of the checkpoint is associated with the appearance of F-actin patches at the bridge entrance that presumably play an important role in bridge stability and prevention of tetraploidy while abscission is halted [46]. Despite rapid advancements, many questions remain unanswered: which exact chromatin defects activate the checkpoint [57]? How are diverse cytokinetic stresses recognized and how do they activate checkpoint kinases? How is F-actin maintained at high levels when the checkpoint is activated in mammalian cells? Are double strand breaks observed when the checkpoint is defective, resulting from nuclear envelope rupture and entry of cytoplasmic nucleases $[45,55,58,59]$ ? Answering some of these questions should help to understand the multiple changes in F-actin and ESCRT-III remodeling that are observed in response to checkpoint activation.

Second, there is an urgent need to confirm in vivo the pathways and mechanisms described within this review in cultured cells. In the few in vivo studies, unexpected results have been 
numerous. For instance, ESCRTs are required for abscission in female germ cells in Drosophila, but not in somatic cells $[60,61]$. Similarly, SEPTIN7 is required for cytokinesis in mouse fibroblasts but not in hematopoietic stem cells [62]. In addition, the septin Peanut is required for cytokinesis in the first (planar) but not the second (orthogonal) division of sensory precursor stem cells in Drosophila [63]. Thus, the filament components described above can be essential or not depending on the context or the organism.

Finally, a recent report exemplified how partial our understanding of late cytokinetic events is. Quite unexpectedly, during the first division of $C$. elegans embryos, no helices could be observed at the abscission site by EM tomography [64]. Instead, ESCRT-III-dependent filamentous helices are unambiguously detected at multiple buds emerging from the midbody. Furthermore, ESCRTs are not essential for abscission in this first division (upon depletion, a slight delay is observed, as opposed to a strong delay found in human cells) [64]. Nevertheless, in both models abscission eventually occurs in most of the cells upon ESCRT-III inactivation. This might be due to residual ESCRT functions or ESCRT component redundancy but might also suggest the existence of an ESCRT-independent mechanism of abscission, which is new territory to be explored in the future.

BOX: Key unanswered questions regarding cytoskeletal remodeling during late cytokinesis

1- Are helices at the abscission site made of ESCRT components?

2- Do ESCRT-dependent helices constrict to completion and trigger abscission?

3- What are the relationships between anillin, septin and ESCRT-dependent helices?

4- How do septins promote ESCRT filament localization?

5- What determines the recruitment of the ESCRT-III pool at the abscission site?

6- How is Rab35 activated and localized to the abscission site?

7- How is F-actin stabilized when the abscission checkpoint is activated?

8- Which pathways are required for abscission in vivo?

9- What are the potential ESCRT-independent mechanisms involved in abscission? 


\section{Acknowledgements}

We thank Ronan Shaughnessy for critical reading of the manuscript. Work from A. Echard's laboratory receives financial support from Institut Pasteur, the Centre National de la Recherche Scientifique (CNRS), the Institut National du Cancer (INCa 2014-1-PLB IO-04), ANR (AbsyStem 15-CE13-0001-02 and CYTOSIGN 16-CE13-004-02) and ASL (Association Syndrome de Lowe). JB has been awarded a doctoral fellowship from the Pasteur Paris University International doctoral program and Carnot-Pasteur MI. CA has been awarded a doctoral fellowship from the Ecole Doctorale "Complexité du Vivant" UPMC and the Ecole Polytechnique. We apologize to those colleagues whose work could not be cited due to space limitations.

\section{References}

Papers of particular interest, published within the period of review, have been highlighted as:

* of special interest

** of outstanding interest

1. Green RA, Paluch E, Oegema K: Cytokinesis in Animal Cells. Annu Rev Cell Dev Biol 2012.

2. D'Avino PP, Giansanti MG, Petronczki M: Cytokinesis in animal cells. Cold Spring Harb Perspect Biol 2015, 7:a015834.

3. Mierzwa B, Gerlich DW: Cytokinetic Abscission: Molecular Mechanisms and Temporal Control. Dev Cell 2014, 31:525-538.

4. Carlton JG, Martin-Serrano J: Parallels between cytokinesis and retroviral budding: a role for the ESCRT machinery. Science 2007, 316:1908-1912.

5. Morita E, Sandrin V, Chung HY, Morham SG, Gygi SP, Rodesch CK, Sundquist WI: Human ESCRT and ALIX proteins interact with proteins of the midbody and function in cytokinesis. Embo J 2007, 26:4215-4227.

6. Stoten CL, Carlton JG: ESCRT-dependent control of membrane remodelling during cell division. Semin Cell Dev Biol 2017.

7. Schoneberg J, Lee IH, Iwasa JH, Hurley JH: Reverse-topology membrane scission by the ESCRT proteins. Nat Rev Mol Cell Biol 2017, 18:5-17.

8. Wollert T, Hurley JH: Molecular mechanism of multivesicular body biogenesis by ESCRT complexes. Nature 2010, 464:864-869. 
9. Echard A, Hickson GR, Foley E, O'Farrell PH: Terminal Cytokinesis Events Uncovered after an RNAi Screen. Curr Biol 2004, 14:1685-1693.

10. Guizetti J, Schermelleh L, Mantler J, Maar S, Poser I, Leonhardt H, Muller-Reichert T, Gerlich DW: Cortical Constriction During Abscission Involves Helices of ESCRT-IIIDependent Filaments. Science 2011, 331:1616-1620.

11. El Amine N, Kechad A, Jananji S, Hickson GR: Opposing actions of septins and Sticky on Anillin promote the transition from contractile to midbody ring. J Cell Biol 2013, 203:487-504.

12. Mostowy S, Cossart P: Septins: the fourth component of the cytoskeleton. Nat Rev Mol Cell Biol 2012, 13:183-194.

13. Kouranti I, Sachse M, Arouche N, Goud B, Echard A: Rab35 regulates an endocytic recycling pathway essential for the terminal steps of cytokinesis. Curr Biol 2006, 16:1719-1725.

14. Estey MP, Di Ciano-Oliveira C, Froese CD, Bejide MT, Trimble WS: Distinct roles of septins in cytokinesis: SEPT9 mediates midbody abscission. J Cell Biol 2010, 191:741749.

15. Kechad A, Jananji S, Ruella $Y$, Hickson GR: Anillin acts as a bifunctional linker coordinating midbody ring biogenesis during cytokinesis. Curr Biol 2012, 22:197203.

* 16. Renshaw MJ, Liu J, Lavoie BD, Wilde A: Anillin-dependent organization of septin filaments promotes intercellular bridge elongation and Chmp4B targeting to the abscission site. Open Biol 2014, 4:130190.

Carefully analyzed the sequential localization of anillin/septins and ESCRT-III at the bridge in human cells, and demonstrated a role for anillin/septins in ESCRT-III recruitment at the abscission site.

17. Green RA, Mayers JR, Wang S, Lewellyn L, Desai A, Audhya A, Oegema K: The midbody ring scaffolds the abscission machinery in the absence of midbody microtubules. $J$ Cell Biol 2013, 203:505-520.

18. Kim MS, Froese CD, Estey MP, Trimble WS: SEPT9 occupies the terminal positions in septin octamers and mediates polymerization-dependent functions in abscission. $J$ Cell Biol 2011, 195:815-826.

19. Johnson ES, Blobel G: Cell cycle-regulated attachment of the ubiquitin-related protein SUMO to the yeast septins. J Cell Biol 1999, 147:981-994.

20. Takahashi $Y$, Iwase M, Konishi M, Tanaka M, Toh-e A, Kikuchi Y: Smt3, a SUMO-1 homolog, is conjugated to $\mathrm{Cdc3}$, a component of septin rings at the mother-bud neck in budding yeast. Biochem Biophys Res Commun 1999, 259:582-587.

* 21. Ribet D, Boscaini S, Cauvin C, Siguier M, Mostowy S, Echard A, Cossart P: SUMOylation of human septins is critical for septin filament bundling and cytokinesis. J Cell Biol 2017, 216:4041-4052.

Demonstrated the SUMOylation of septins in human cells, identified non-SUMOylable mutants and revealed the role of SUMOylation in preventing the formation of abnormal septin bundles that perturb cytokinesis. 
22. Elia N, Sougrat R, Spurlin TA, Hurley JH, Lippincott-Schwartz J: Dynamics of endosomal sorting complex required for transport (ESCRT) machinery during cytokinesis and its role in abscission. Proc Natl Acad Sci U S A 2011, 108:4846-4851.

23. Schiel JA, Simon GC, Zaharris C, Weisz J, Castle D, Wu CC, Prekeris R: FIP3-endosomedependent formation of the secondary ingression mediates ESCRT-III recruitment during cytokinesis. Nat Cell Biol 2012, 14:1068-1078.

24. Crowell EF, Gaffuri AL, Gayraud-Morel B, Tajbakhsh S, Echard A: Midbody remnant engulfment after cytokinesis abscission in mammalian cells. J Cell Sci 2014, 127:3840-3851.

25. Sherman S, Kirchenbuechler D, Nachmias D, Tamir A, Werner S, Elbaum M, Elia N: Resolving new ultrastructural features of cytokinetic abscission with soft-X-ray cryo-tomography. Sci Rep 2016, 6:27629.

** 26. Mierzwa BE, Chiaruttini N, Redondo-Morata L, von Filseck JM, Konig J, Larios J, Poser I, Muller-Reichert T, Scheuring S, Roux A, et al.: Dynamic subunit turnover in ESCRT-III assemblies is regulated by Vps4 to mediate membrane remodelling during cytokinesis. Nat Cell Biol 2017, 19:787-798.

Revealed a continous recruitment of VPS4 and ESCRT-III components during bridge maturation in human cells, demonstrated the essential role of ESCRT-III turnover by VPS4 for ESCRT accumulation and succesful abscission in vivo and for ESCRT spiral constriction in vitro.

27. Atilla-Gokcumen GE, Muro E, Relat-Goberna J, Sasse S, Bedigian A, Coughlin ML, GarciaManyes S, Eggert US: Dividing cells regulate their lipid composition and localization. Cell 2014, 156:428-439.

28. Cauvin C, Echard A: Phosphoinositides: Lipids with informative heads and mastermind functions in cell division. Biochim Biophys Acta 2015, 1851:832-843.

29. Lee HH, Elia N, Ghirlando R, Lippincott-Schwartz J, Hurley JH: Midbody targeting of the ESCRT machinery by a noncanonical coiled coil in CEP55. Science 2008, 322:576-580.

* 30. Christ L, Wenzel EM, Liestol K, Raiborg C, Campsteijn C, Stenmark H: ALIX and ESCRTI/II function as parallel ESCRT-III recruiters in cytokinetic abscission. J Cell Biol 2016, 212:499-513.

Found that two pathways, one depending on TSG101/ESCRT-II and the other depending on ALIX, act in parallel to recruit ESCRT-III at the intercellular bridge in mammalian cells, and revealed the specific role of the ALIX pathway in the NoCut checkpoint.

31. Sun S, Sun L, Zhou X, Wu C, Wang R, Lin SH, Kuang J: Phosphorylation-Dependent Activation of the ESCRT Function of ALIX in Cytokinetic Abscission and Retroviral Budding. Dev Cell 2016, 37:581.

32. Elia N, Fabrikant G, Kozlov MM, Lippincott-Schwartz J: Computational Model of Cytokinetic Abscission Driven by ESCRT-III Polymerization and Remodeling. Biophys J 2012, 102:2309-2320.

33. Lafaurie-Janvore J, Maiuri P, Wang I, Pinot M, Manneville JB, Betz T, Balland M, Piel M: ESCRT-III assembly and cytokinetic abscission are induced by tension release in the intercellular bridge. Science 2013, 339:1625-1629. 
34. Adell MAY, Migliano SM, Upadhyayula S, Bykov YS, Sprenger S, Pakdel M, Vogel GF, Jih G, Skillern W, Behrouzi R, et al.: Recruitment dynamics of ESCRT-III and Vps4 to endosomes and implications for reverse membrane budding. Elife 2017, 6.

35. Connell JW, Lindon C, Luzio JP, Reid E: Spastin couples microtubule severing to membrane traffic in completion of cytokinesis and secretion. Traffic 2009, 10:42-56.

36. Yang D, Rismanchi N, Renvoise B, Lippincott-Schwartz J, Blackstone C, Hurley JH: Structural basis for midbody targeting of spastin by the ESCRT-III protein CHMP1B. Nat Struct Mol Biol 2008, 15:1278-1286.

* 37. Vietri M, Schink KO, Campsteijn C, Wegner CS, Schultz SW, Christ L, Thoresen SB, Brech A, Raiborg C, Stenmark H: Spastin and ESCRT-III coordinate mitotic spindle disassembly and nuclear envelope sealing. Nature 2015, 522:231-235.

Drew an interesting parallel between the molecules involved in cytokinesis and nuclear envelope reformation.

38. Schiel JA, Park K, Morphew MK, Reid E, Hoenger A, Prekeris R: Endocytic membrane fusion and buckling-induced microtubule severing mediate cell abscission. J Cell Sci 2011, 124:1411-1424.

39. Dambournet $D$, Machicoane $M$, Chesneau L, Sachse $M$, Rocancourt $M$, El Marjou A, Formstecher E, Salomon R, Goud B, Echard A: Rab35 GTPase and OCRL phosphatase remodel lipids and F-actin for successful cytokinesis. Nat Cell Biol 2011, 13:981-988.

** 40. Fremont S, Hammich H, Bai J, Wioland H, Klinkert K, Rocancourt M, Kikuti C, Stroebel $\mathrm{D}$, Romet-Lemonne G, Pylypenko O, et al.: Oxidation of F-actin controls the terminal steps of cytokinesis. Nat Commun 2017, 8:14528.

Revealed the first connection between oxidoreduction and cell division, showed that Rab35 GTPase activates MICAL1 enzymatic activity toward F-actin depolymerization in vitro, found a role for Rab35 in recruiting MICAL1 at the abscission site for successful abscission.

41. Cauvin C, Rosendale M, Gupta-Rossi N, Rocancourt M, Larraufie P, Salomon R, Perrais D, Echard A: Rab35 GTPase Triggers Switch-like Recruitment of the Lowe Syndrome Lipid Phosphatase OCRL on Newborn Endosomes. Curr Biol 2016, 26:120-128.

42. Hung RJ, Pak CW, Terman JR: Direct redox regulation of F-actin assembly and disassembly by Mical. Science 2011, 334:1710-1713.

43. Fremont $S$, Romet-Lemonne G, Houdusse A, Echard A: Emerging roles of MICAL family proteins - from actin oxidation to membrane trafficking during cytokinesis. J Cell Sci 2017, 130:1509-1517.

44. Fremont S, Echard A: Membrane Traffic and Cytokinesis: The Final Cut under review.

45. Norden C, Mendoza M, Dobbelaere J, Kotwaliwale CV, Biggins S, Barral Y: The NoCut pathway links completion of cytokinesis to spindle midzone function to prevent chromosome breakage. Cell 2006, 125:85-98.

46. Steigemann P, Wurzenberger C, Schmitz MH, Held M, Guizetti J, Maar S, Gerlich DW: Aurora B-mediated abscission checkpoint protects against tetraploidization. Cell 2009, 136:473-484.

47. Mackay DR, Makise M, Ullman KS: Defects in nuclear pore assembly lead to activation of an Aurora B-mediated abscission checkpoint. J Cell Biol 2010, 191:923-931. 
48. Mathieu J, Cauvin C, Moch C, Radford SJ, Sampaio P, Perdigoto CN, Schweisguth F, Bardin AJ, Sunkel CE, McKim K, et al.: Aurora B and cyclin B have opposite effects on the timing of cytokinesis abscission in Drosophila germ cells and in vertebrate somatic cells. Dev Cell 2013, 26:250-265.

49. Mackay DR, Ullman KS: ATR and a Chk1-Aurora B pathway coordinate postmitotic genome surveillance with cytokinetic abscission. Mol Biol Cell 2015, 26:2217-2226.

50. Nahse V, Christ L, Stenmark H, Campsteijn C: The Abscission Checkpoint: Making It to the Final Cut. Trends Cell Biol 2017, 27:1-11.

51. Carlton JG, Caballe A, Agromayor M, Kloc M, Martin-Serrano J: ESCRT-III governs the Aurora B-mediated abscission checkpoint through CHMP4C. Science 2012, 336:220225.

52. Capalbo L, Montembault E, Takeda T, Bassi ZI, Glover DM, D'Avino PP: The chromosomal passenger complex controls the function of endosomal sorting complex required for transport-III Snf7 proteins during cytokinesis. Open Biol 2012, 2:120070.

53. Capalbo L, Mela I, Abad MA, Jeyaprakash AA, Edwardson JM, D'Avino PP: Coordinated regulation of the ESCRT-III component CHMP4C by the chromosomal passenger complex and centralspindlin during cytokinesis. Open Biol 2016, 6.

54. Thoresen SB, Campsteijn C, Vietri M, Schink KO, Liestol K, Andersen JS, Raiborg C, Stenmark H: ANCHR mediates Aurora-B-dependent abscission checkpoint control through retention of VPS4. Nat Cell Biol 2014.

* 55. Caballe A, Wenzel DM, Agromayor M, Alam SL, Skalicky JJ, Kloc M, Carlton JG, Labrador L, Sundquist WI, Martin-Serrano J: ULK3 regulates cytokinetic abscission by phosphorylating ESCRT-III proteins. Elife 2015, 4:e06547.

* 56. Petsalaki E, Zachos G: Clks 1, 2 and 4 prevent chromatin breakage by regulating the Aurora B-dependent abscission checkpoint. Nat Commun 2016, 7:11451.

References 55 and 56 revealed new kinases involved in the NoCut checkpoint in mammalian cells.

*57. Amaral N, Vendrell A, Funaya C, Idrissi FZ, Maier M, Kumar A, Neurohr G, Colomina N, Torres-Rosell J, Geli MI, et al.: The Aurora-B-dependent NoCut checkpoint prevents damage of anaphase bridges after DNA replication stress. Nat Cell Biol 2016, 18:516-526.

Revealed that not all kinds of DNA bridges activate the NoCut checkpoint in budding yeast, and that the checkpoint specifically promotes viability in the presence of chromatin bridges resulting from replication stress.

58. Maciejowski J, Li Y, Bosco N, Campbell PJ, de Lange T: Chromothripsis and Kataegis Induced by Telomere Crisis. Cell 2015, 163:1641-1654.

59. Raab M, Gentili M, de Belly $H$, Thiam HR, Vargas $P$, Jimenez AJ, Lautenschlaeger $F$, Voituriez R, Lennon-Dumenil AM, Manel N, et al.: ESCRT III repairs nuclear envelope ruptures during cell migration to limit DNA damage and cell death. Science 2016, 352:359-362.

* 60. Matias NR, Mathieu J, Huynh JR: Abscission is regulated by the ESCRT-III protein shrub in Drosophila germline stem cells. PLoS Genet 2015, 11:e1004653. 
* 61. Eikenes AH, Malerod L, Christensen AL, Steen CB, Mathieu J, Nezis IP, Liestol K, Huynh JR, Stenmark H, Haglund K: ALIX and ESCRT-III coordinately control cytokinetic abscission during germline stem cell division in vivo. PLoS Genet 2015, 11:e1004904. References 60 and 61 demonstrated for the first time a requirement of ESCRT-III in cytokinesis in animal cells in vivo, in female germ line stem cells.

62. Menon MB, Sawada A, Chaturvedi A, Mishra P, Schuster-Gossler K, Galla M, Schambach A, Gossler A, Forster R, Heuser M, et al.: Genetic deletion of SEPT7 reveals a cell type-specific role of septins in microtubule destabilization for the completion of cytokinesis. PLoS Genet 2014, 10:e1004558.

* 63. Founounou N, Loyer N, Le Borgne R: Septins regulate the contractility of the actomyosin ring to enable adherens junction remodeling during cytokinesis of epithelial cells. Dev Cell 2013, 24:242-255.

Revealed that the role of septins is context-dependent and are required for planar but not orthogonal division in the cell lineage leading to the formation of sensory organs.

** 64. Konig J, Frankel EB, Audhya A, Muller-Reichert T: Membrane remodeling during embryonic abscission in Caenorhabditis elegans. J Cell Biol 2017, 216:1277-1286.

Revealed that in the absence of ESCRT function, cytokinetic abscission was only sightly delayed in C. elegans embryos, and that ESCRT-dependent helices were not observed during cytokinesis (as previsouly found in human cells), suggesting that ESCRTindependent mechanisms of abscission may exist. 


\section{A. Septin SUMOylation}

Direct effect of SUMOylation on filament-filament interactions ?

Regulation of filament-filament interactions via SUMO-dependent binding factors?

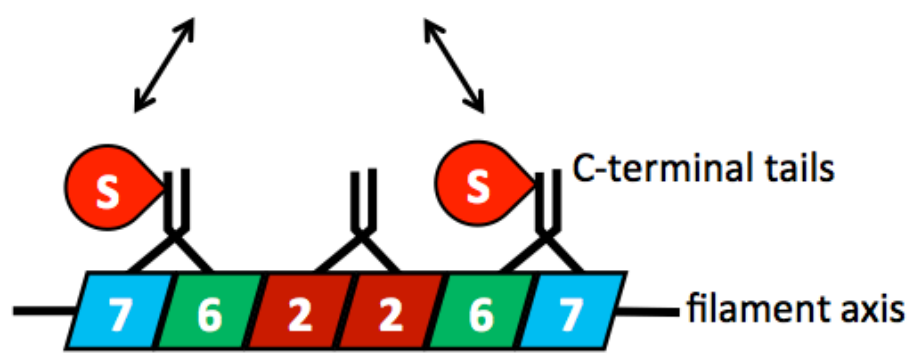

Septin filament unit

\section{B. Actin oxidation}

Barbed-end

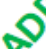

Pointed-end

MICAL1 binds

to F-actin
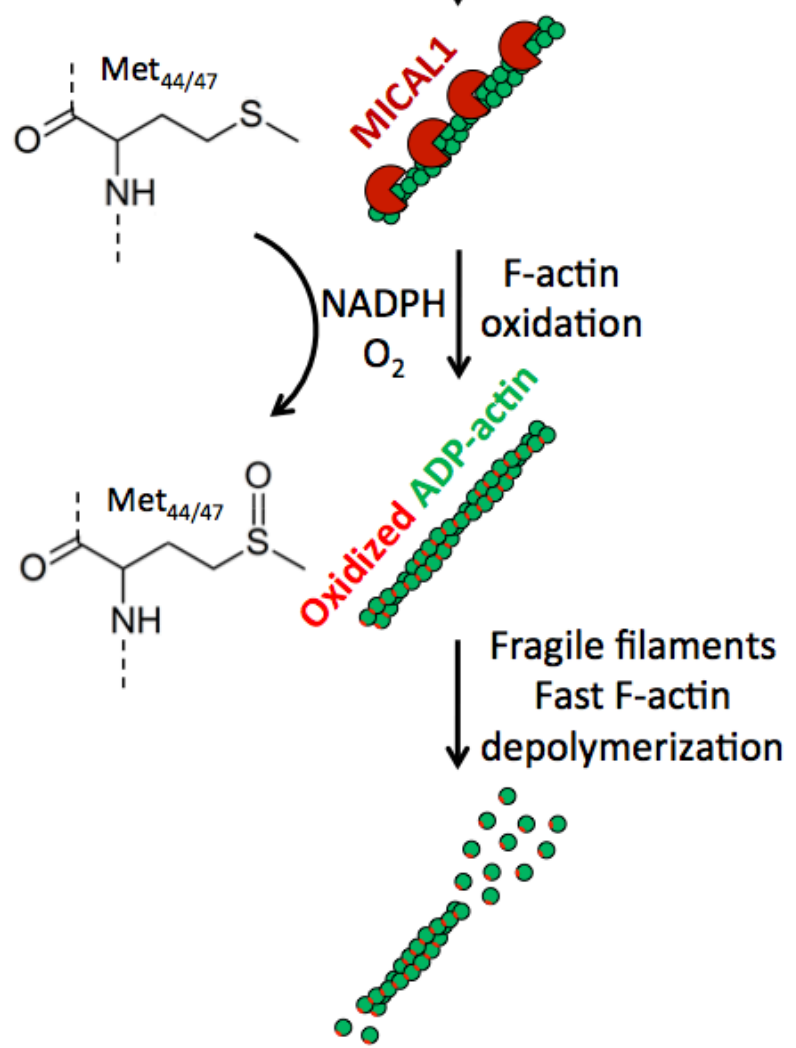

Figure 1: Two post-translational modifications of cytoskeletal elements recently involved in cytokinesis. (A) SUMOylation of Septin 6, 7 and 11 on their C-terminal tails prevents abnormal bundling of Septin filaments and destabilizes cytokinetic bridges in human cells. SUMOylation could either directly affect filament-filament interactions or regulate filament bundling through SUMO-dependent binding factors. (B) Actin oxidation of methionine residues (Met44 and M47) into methionine sulfoxide induces fast F-actin depolymerization. The reaction involves the enzyme MICAL1, $\mathrm{O}_{2}$ and NADPH as a cofactor. MICAL1-dependent actin oxidation is required for correct localization of ESCRT-III at the secondary ingression site and for normal timing of abscission in human cells. 

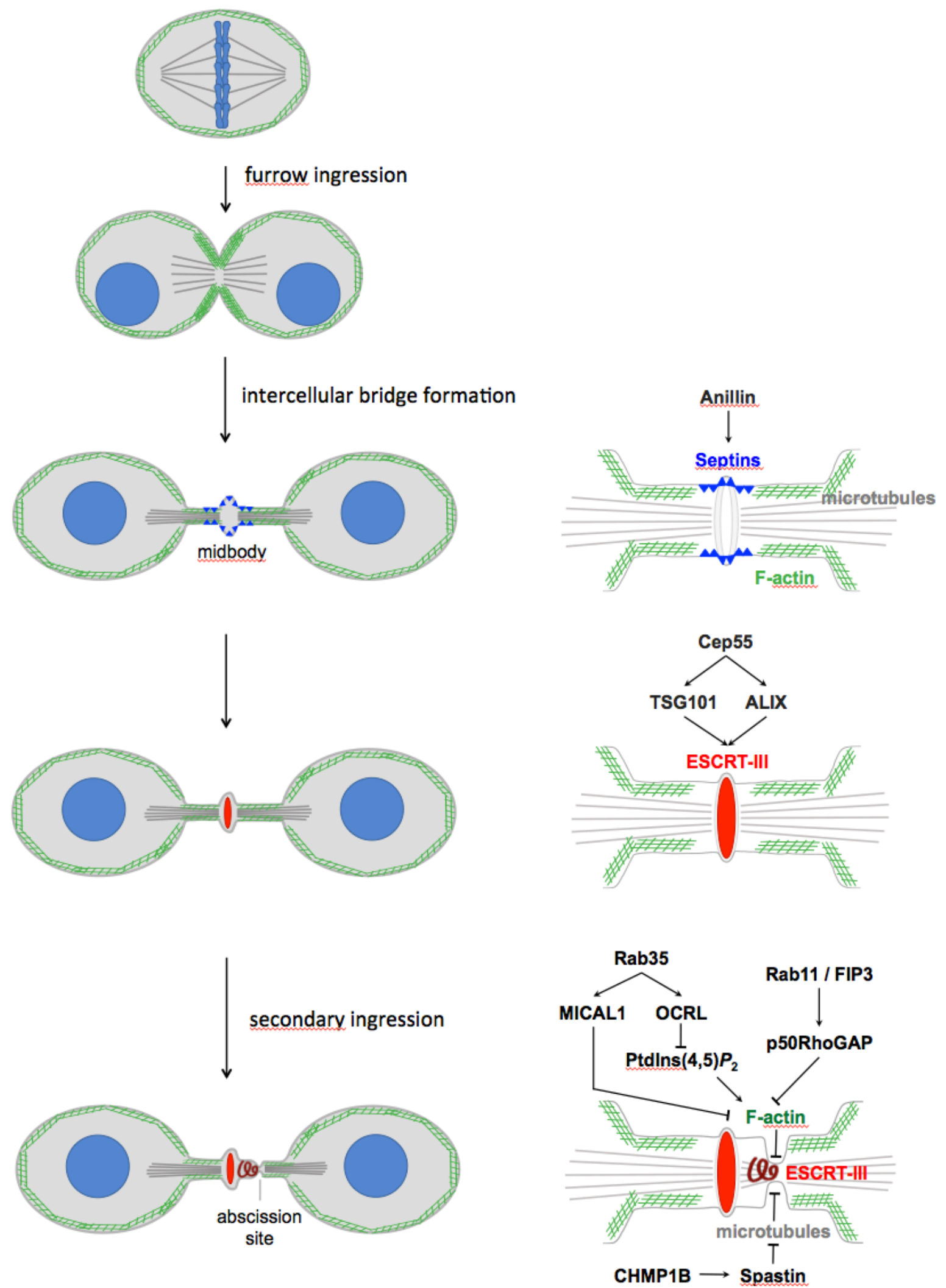

Figure 2: Mechanisms controlling cytoskeletal remodeling in the post-furrowing steps of cytokinesis. Septins, F-actin, microtubules and ESCRT-III filaments are indicated in blue, green, grey and red, respectively. Note that ESCRT-III recruitment and constriction at the abscission site require clearance of both microtubules and F-actin. 